\title{
INTELLIGENT OTN IN THE TLC OPERATOR INFRASTRUCTURES
}

Ovidio Michelangeli WIND Telecomunicazioni S.p.A.Via C.G.Viola 48 Roma ovidio.michelangeli@mail.wind.it, Alberto Mittoni: WIND Telecomunicazioni S.p.A.Via C.G.Viola 48 Roma alberto.mittoni@mail.wind.it.

\footnotetext{
Abstract: The operators are today facing the migration of their transport infrastructures toward intelligent optical layer by introducing in the core optical switches based on GMPLS/ASON protocol. The paper describes the advantages can be achieved by using the next generation Optical Platforms by comparing the ring and mesh network.

Network restorations in the distributed and centralised control plane are also compared by showing some lab test results. Finally are outlined the current limitations and the issues still open to be solved by the industry and the standard bodies.
}

\section{INTRODUCTION}

The continued growth in high-speed Internet applications will drive the backbone transport network migration for many TLC operators in the coming years. The existing infrastructures designed on ring topology are not more efficient to support the traffic demand due to the fixed and mobile broadband services. The technological platform in the core are moving toward next generation network based on packet switching rather than circuit switching and the traffic behaviour changes from the traditional voice traffic (constant) to Internet traffic (instantaneous with large peak ). 


\section{DRIVERS TO MIGRATE THE BACKBONE TRANSPORT NETWORK AND OPERATOR REQUIREMENTS}

The traffic demand for telecommunications services increases the transmission flows in long distance transport network. Fixed and mobile services based on multimedia applications will require an additional bandwidth to the transport network.

The Operators ask for new mesh layer having the following functionality:

- Real time Bandwidth allocation: Network solutions have to cope with uncertainty in bandwidth needs.

- Faster Provisioning: As new services come into effect, clients demand faster access to these services.

- Seamless migration to an Intelligent Transport Network: Network Service Provider with existing SDH or DWDM networks need to protect their investment with a smooth migration path of their networks to the optical layer.

- Service differentiation: In a highly competitive environment, prices become lower and equalized. The Network Service Provider that can offer a wider array of services becomes the winner.

- Migration to new services support: new services are in demand by most clients who want to benefit from the ITN new features (Ethernet, transparent wavelength services, etc.).

$\checkmark$ Optical Virtual Private Networks

$\checkmark \quad$ Transparent Wavelengths leasing

$\checkmark$ Ethernet Services

$\checkmark$ Bandwidth adapting Data Service

- Operational cost reduction: More and more complex networks start to take a toll on maintenance, provisioning, performance control, etc.

TLC Operators are demanding ways to achieve that all these operational issues do not translate into higher costs.

\section{GMPLS/ASON ARCHITECTURE}

The GMPLS/ASON architecture [2] is based on mesh topology that is more suitable for large network with different traffic protection requirements. [2,3] It requires less capacity and then are cheaper than ring topology. Figure 2 illustrates the GMPLS/ASON network architecture that is divided in three main domains:

- Control plane: performs the call and connection control functions. Through signalling, it sets up and releases connections, and may restore a connection in case of a failure. 
- The management plane: performs management functions: fault, configuration, accounting, performance and security.

- The transport plane: provides flow transfer for user information from one location to another. It can also provide flow transfer for some control and network management information. The transport plane is layered as it is equivalent to the Transport Network defined in ITU Rec. G.805.

ASON/GMPLS is a transport network that, via control plane, can provide automatic network resource and state information discovery. Furthermore it allow continue monitoring of the network and the connected links. It can set up and delete connection.

Different regulatory and organisation bodies have been involved to develop the new intelligent optical transport network. The most important are the following:

- $\quad$ IETF (Internet Engineering Task Force) -> GMPLS

- ITU-T (International Telecommunication Union-Transmissions)->ASON framework

- $\quad$ OIF (Optical Internetworking Forum) $\rightarrow>$ OIF-UNI/NNI

Other organisations (such as ANSI, MPLS Forum) still take part in the GMPLS standardisation activity, but the ones cited above are the most important.

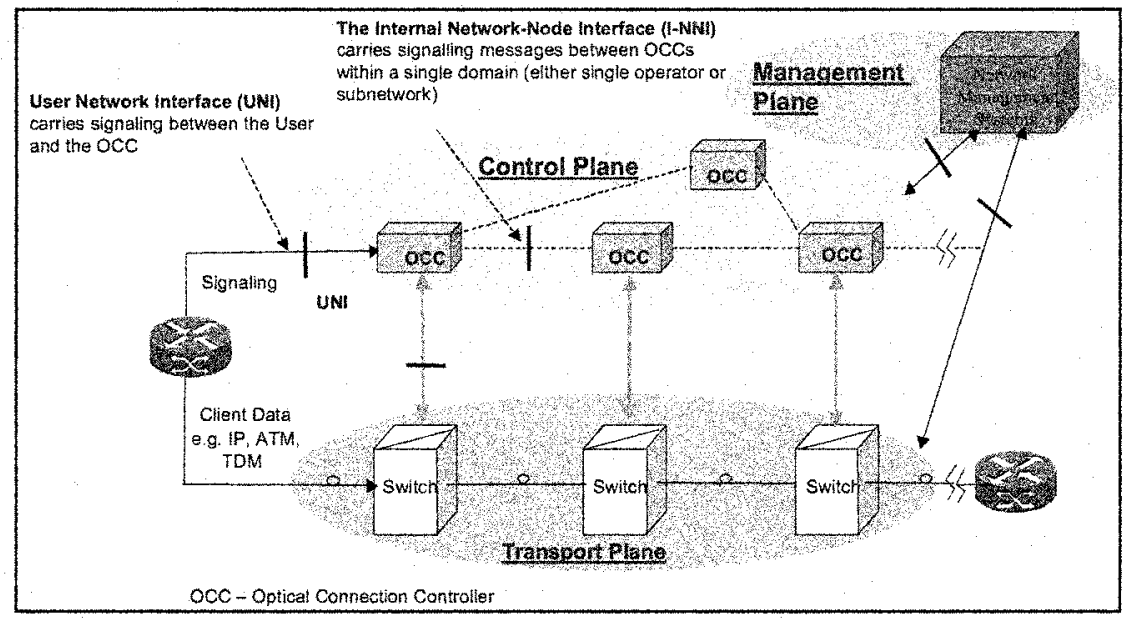

Figure 2: GMPLS/ASON network architecture

\section{ADVANTAGES FOR THE TLC OPERATOR}

The new architecture can offer different advantages for the network operators need. The automatic services in the GMPLS/ASON Architecture allow to reduce OPEX (manual operations are limited) in terms of provisioning and management aspects. With automatic provisioning, request might be directly sent by end user 


\section{8}

and managed by ASON control plane (new services for end user, immediate implementation of customer order). The operation of the network is cheaper and have a more efficient network management (faults are directly handled by control plane and traffic is immediately restored.). below are described the further advantages for the Network operators:

- Network Availability (in case of multiple faults)

- CAPEX reduction

- Network flexibility

- New revenue generating services (Value added services improve revenues)

\subsection{Network availability}

Figure 3 shows the Availability versus MTTR. By increasing the MTTR (i.e increasing of multiple faults in the network) the restoration improve the availability figures.

In the multiple rings an equipment fault cause an automatic switch in the protection path. As consequence (for instance in the maintenance works) the path is not protected.

In the restoration the control plane in case of path failure can dynamically find a protection path where the traffic can be rerouted. In this case the system remain always protected (until there are spare path) even if there are multiple faults (for instance some links are de activated for maintenance purposes)

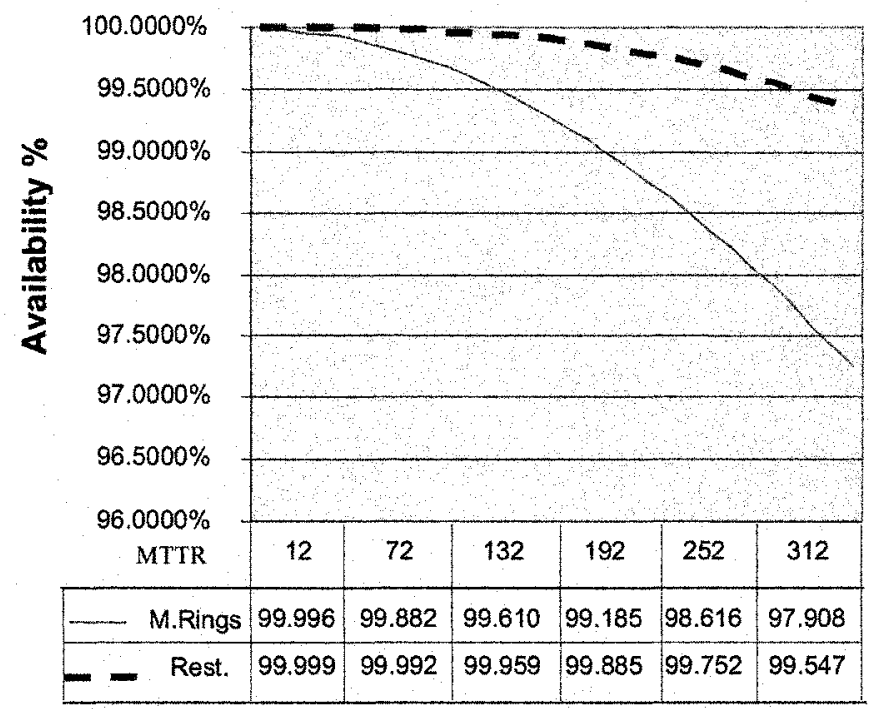

Figure 3: Availability comparison between mutiple ring and mesh network with restoration. 


\subsection{Capex reduction}

TLC operators are now concentrated at the core business. They are today driven by business case to deploy new technological platform in their network to minimize CAPEX (maximize the existing infrastructure) and reduce OPEX (simplified service configuration and provisioning). A simple Capex reduction is not more sufficient. New Investment should provide not only revenue but also a reduction of operational expenses. Before to implement new infrastructure in their networks, TLC operator prefer to make a business case in order to be sure that new technology can respond their need and optimising their investment.

WIND achieved a case study to evaluate the total cost of its transport backbone network by considering a time frame of 3 years. As input the existing network topology and the traffic matrix for three years has been provided. As output the total network cost in two different scenario has been evaluated:

a) Migration of the existing network following the traditional ring topology;

b) Migration of the existing network with the introduction of a mesh layer based on GMPLS/ASON architecture.

\subsection{Case study results}

In the following are showed the main results.

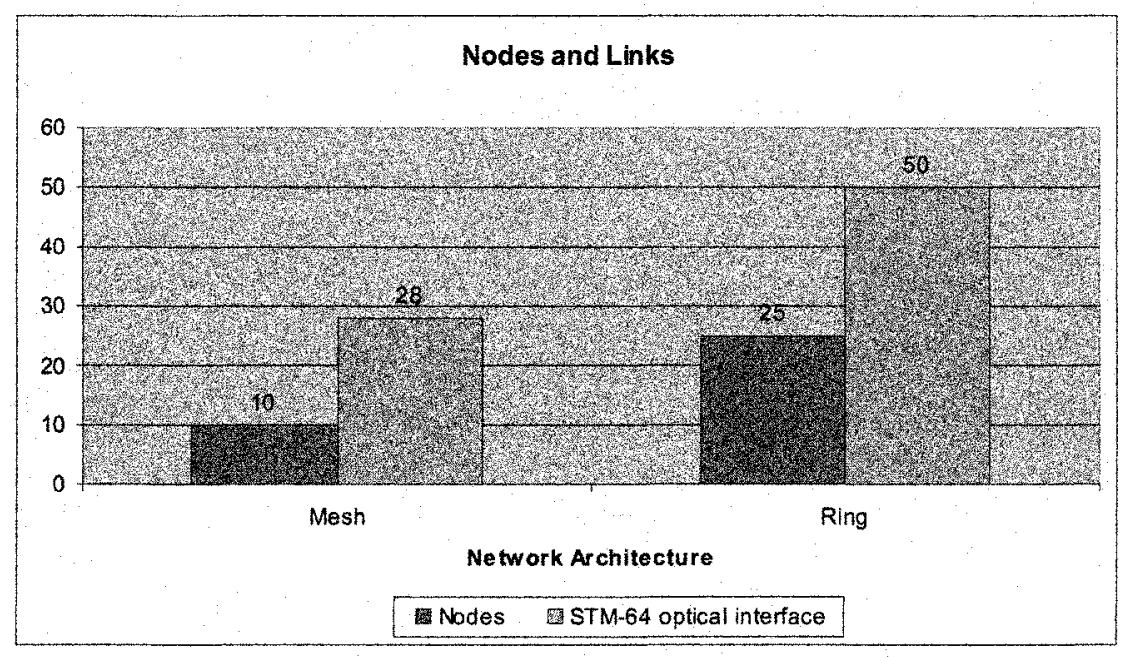

Figure 4: Number of nodes and STM 64 optical interfaces for ring and mesh network with restoration

With reference to Figure 4 the introduction of mesh network with restoration allows to save DWDM links and the number of the equipment in the nodes. The 
restoration technique allows to share the transmission resources dedicated to the protection and then decreasing the number of $10 \mathrm{Gbps}$ transponder in the network. The mesh networks with restoration allow to save $70 \%$ of capacity for protection. In Figure 5 is illustrated the CAPEX with its main item: Electro - Optical Cross Connect, Network Management System and the DWDM links.

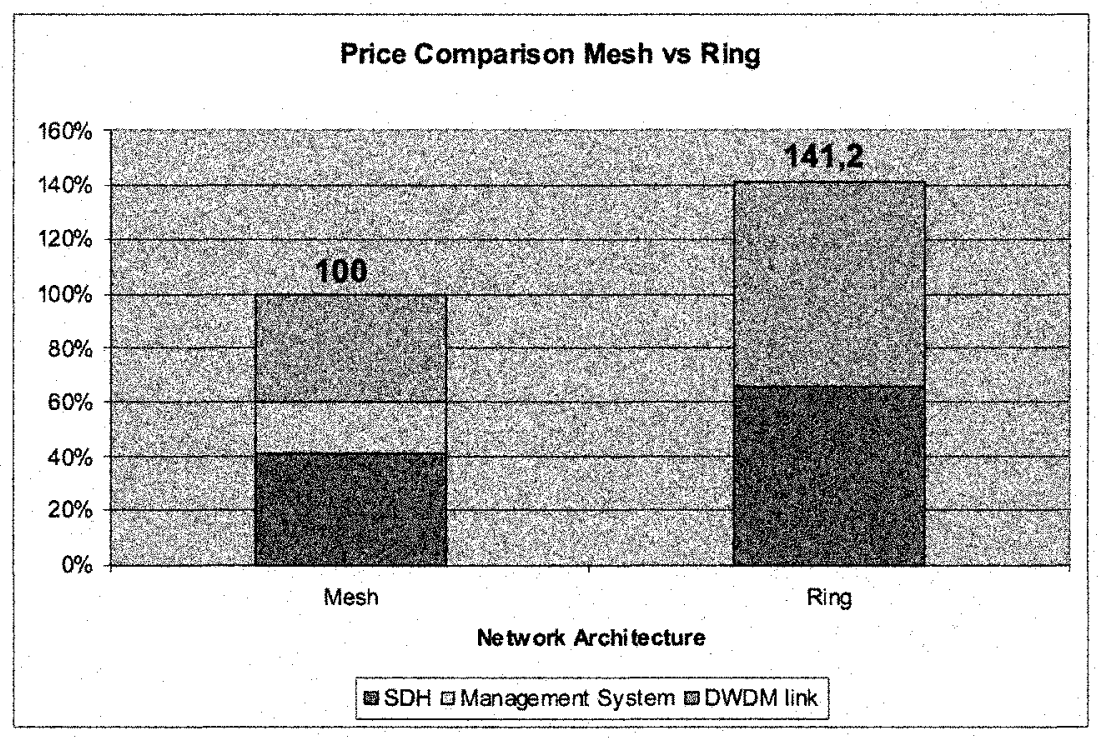

Figure 5 : Comparison between CAPEX for Ring and meshed network with restoration

\subsection{Network Flexibility}

The network upgrade in the mesh network is very simple because is sufficient to add only $\mathrm{I} / \mathrm{O}$ interface in the equipment. In the ring topology the network upgrading require to add a new network element. Typically, in case of upgrade, the number of adding section required in the mesh network with restoration are less than $50 \%$ respect to those necessary on ring network.

\subsection{New revenue generating services}

The GMPLS/ASON architecture will allow the operators to offer new revenue generating services. The contract negotiations will move from individual connections to service level agreement. The end to end connectivity will be automated and will be Client-driven.

In addition services such as bandwidth on demand, optical virtual private network, differentiated services based on QoS will be easily provided to the customers. 


\section{WIND TRANSPORT NETWORK MIGRATION}

On the basis of the business case results WIND decided to introduce a mesh network layer to gather only very big nodes and to transport high level traffic (STM-N $\mathrm{N} \geq 1$ ) as show in Figure 6.

The ring network will be used for national/regional area and for transport of low level traffic ( $\mathrm{VCl} 2$ and $\mathrm{VC} 3$ ). The new layer is realised by a electrical optical - switch at high capacity connected with other nodes (from 2 to 5) via DWDM link at $10 \mathrm{Gbps}$. The network protection will be configurable for each path with different protection level by using the resources dedicated to the shared protection. Control Plane will be centralised and it will migrate in distributed configuration in the medium term.

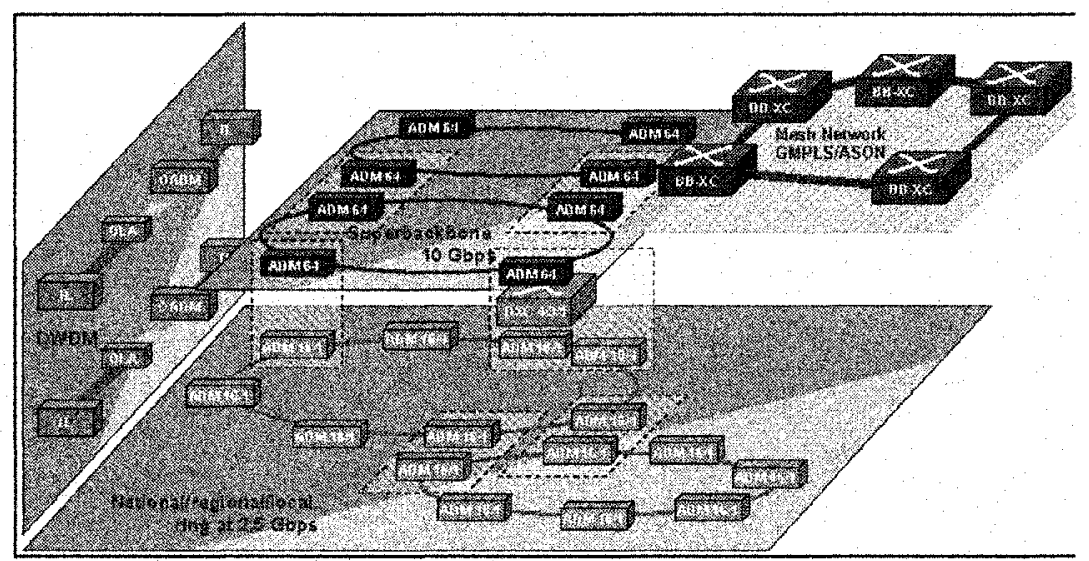

Figure 6: Wind backbone transport network evolution

\subsection{Test results}

Wind achieved in the vendor laboratories some functional test in order to verify the main Technical performance. Table 1 shows the restoration time for multiple faults in tree different restoration technology: one based on the distributed control plane, one based on the centralised control plane and the last one based on Pre Planned configuration where the restoration is distributed and the provisioning is centralised. 
Table 1: Restoration time versus distributed and Pre-Planned control plane.

\begin{tabular}{|l|l|l|l|}
\hline $\begin{array}{l}\text { Restoration } \\
\text { Technology }\end{array}$ & \multicolumn{2}{|c|}{ On The flight } & Pre-Planned \\
\hline Control Plane & Distributed & Centralised & $\begin{array}{l}\text { Restoration : distributed, } \\
\text { Provisioning: Centralised }\end{array}$ \\
\hline $\begin{array}{l}\text { Restoration time } \\
\text { for multiple paths }\end{array}$ & $500-1000$ msec. & $300-500$ msec. & $500-850 \mathrm{msec}$. \\
\hline
\end{tabular}

\section{CONCLUSION AND OPEN ISSUES}

The all-optical core vision is becoming a reality. Optical Intelligent networks are viable and will see a gradual expansion in the operator backbone transport network in the coming years. The main issues to be solved by the equipment manufacturers and regulatory bodies is to standardise the control plane protocol still proprietary, in order to allow the multi - vendor interoperability. The restoration algorithm is the key to design the mesh intelligent network. Its functionality should cover two important aspects: Obtaining a restoration time from $200 \mathrm{msec}$ to 1 second in order to avoid conflict with the protection mechanism of the client layers; be able to minimise the DWDM links in the mesh network to reduce the investment of the Telecom Operators.

\section{REFERENCES}

[1] Ovidio Michelangeli, "Choosing between OEO and OOO solutions in the core and making a business case for DWDM in the MAN at the edge level" Optical Switching Summit 30-31 January 2002.

[2] E.Mannie et al.,"Generalized Multi-protocol label Switching (GMPLS) architecture “ Internet Draft <draft-ietf-ccamp-gmpls-architecture-05.txt>, Mar.2003

[3] Ovidio Michelangeli , Evolving WIND's Transmission Core Network towards an intelligent meshed network based on GMPLS/ASON. IIR conference "Evolving SDH to support data services. London 3oth March- $1^{\text {st }}$ April

[4] Ovidio Michelangeli; Alberto Mittoni; "Inserimento di reti ottiche magliate nella rete di backbone" Riva del Garda - Fotonica 2003 